\title{
Hyperattenuations on flat-panel computed tomography after successful recanalization of mechanical thrombectomy for anterior circulation occlusion
}

\author{
Yeongu Chung ${ }^{\wedge}$, Youngoh Bae ${ }^{1}$, Chang Eui Hong ${ }^{1}$, Yu Sam Won ${ }^{1}$, Jang-Hyun Baek ${ }^{2}$, Pil-Wook Chung², \\ Myung Sub Kim ${ }^{3}$, Myung Ho Rho ${ }^{3}$ \\ ${ }^{1}$ Department of Neurosurgery, Kangbuk Samsung Hospital, Sungkyunkwan University School of Medicine, Seoul, Republic of Korea; ${ }^{2}$ Department \\ of Neurology, Kangbuk Samsung Hospital, Sungkyunkwan University School of Medicine, Seoul, Republic of Korea; ${ }^{3}$ Department of Radiology, \\ Kangbuk Samsung Hospital, Sungkyunkwan University School of Medicine, Seoul, Republic of Korea
}

Contributions: (I) Conception and design: MH Rho; (II) Administrative support: Y Chung, MH Rho; (III) Provision of study materials or patients: YS Won, JH Baek, PW Chung; (IV) Collection and assembly of data: Y Chung, CE Hong; (V) Data analysis and interpretation: Y Chung, Y Bae, MS Kim; (VI) Manuscript writing: All authors; (VII) Final approval of manuscript: All authors.

Correspondence to: Myung Ho Rho. Departments of Radiology, Kangbuk Samsung Hospital, Sungkyunkwan University School of Medicine, 29 Saemunan-ro, Jongro-gu, Seoul 03181, Republic of Korea. Email: mh.rho@samsung.com.

Background: To evaluate intraparenchymal hyperattenuation (IPH) on flat-panel computed tomography (FPCT) findings and their clinical usefulness for predicting prognosis after successful mechanical thrombectomy (MT) for acute occlusion of anterior circulation.

Methods: A retrospective review was conducted for 158 consecutive patients undergoing mechanical thrombectomy during the last six years. After excluding those with posterior circulation occlusion or incomplete recanalization and those without FPCT, 82 patients were finally included. Immediate postprocedural IPH on FPCT was categorized into four patterns (none, striatal, cortical, or combined pattern). Follow-up magnetic resonance images or CT scans after 48 hours from MT were analyzed according to FPCT findings. The existence of hemorrhagic transformation, intracerebral hemorrhage, and brain swelling was evaluated. Functional clinical outcomes were accessed with post-procedural 3-month modified Rankin scales (mRS).

Results: Of 82 patients, 34 patients were found to have IPH (16 with a striatal pattern, 8 with a cortical pattern, and 10 with a combined pattern). Hemorrhagic complication $(\mathrm{P}<0.001)$, brain swelling $(\mathrm{P}<0.001)$, and poor $\mathrm{mRS}$ scores $(\mathrm{P}=0.042)$ showed significant differences according to IPH patterns. Multivariate logistic regression analysis revealed that the presence of a striatal pattern $(\mathrm{OR}: 13.26, \mathrm{P}<0.001)$, cortical pattern (OR: 11.61, $\mathrm{P}=0.009)$, and combined pattern (OR: 45.34, $\mathrm{P}<0.001)$ independently predicted hemorrhagic complications. The location of the occlusion (OR: 4.13, $\mathrm{P}=0.034)$, cortical pattern (OR: 5.94, $\mathrm{P}=0.039)$, and combined pattern (OR: 39.85, $\mathrm{P}=0.001)$ predicted brain swelling. Age (OR: 1.07, $\mathrm{P}=0.006)$ and the presence of a combined pattern (OR: 10.58, $\mathrm{P}=0.046)$ predicted poor clinical outcomes.

Conclusions: FPCT is a rapid and effective tool for a prompt follow-up just after MT to predict prognosis. Those with striatal patterns showed relatively good clinical outcomes despite significant hemorrhage. Cortical IPH patterns independently predicted a high rate of post-procedural hemorrhage or brain swelling. Combined pattern is a strong predictor for both radiologic and poor clinical outcomes.

Keywords: Computed tomography (CT); ischemic stroke; thrombectomy

\footnotetext{
^ ORCID: 0000-0001-9139-0988.
} 
Submitted Mar 22, 2021. Accepted for publication Aug 09, 2021.

doi: 10.21037/qims-21-322

View this article at: https://dx.doi.org/10.21037/qims-21-322

\section{Introduction}

Cerebral intraparenchymal hyperattenuation (IPH) is frequently recognized on computed tomography (CT) scans after mechanical thrombectomy (MT) for the treatment of ischemic stroke. It has been described in $23 \%$ to $84 \%$ of patients (1). As such, IPH observed either on conventional multidetector CT or flat-panel CT (FPCT) immediately following MT can provide prognostic information about the eventual volume of infarction $(1,2)$. Differentiation of petechial hemorrhage and HT (hemorrhagic transformation) from contrast staining is important for deciding the following antithrombotic therapy. Follow-up CT scan is needed at least 19-24 hours after endovascular therapy (3-6). However, this is not always easy. The presumed mechanism of contrast staining is leakage of the contrast media into the parenchymal extracellular space in areas with ischemiainduced breakdown of the blood-brain barrier (BBB) and basal lamina, BBB disruption due to chemotoxicity or reperfusion injuries following recanalization, or impaired autoregulation/luxury perfusion (7). The significance of IPH after MT is quite variable, ranging from asymptomatic to poor clinical outcome (8-10). The high density of brain parenchyma in patients with acute ischemic stroke after MT most often evolves into cerebral infarction but rarely into hemorrhages (2). The clinical usefulness of IPH and other findings on FPCT immediately after MT has been rarely reported to the best of our knowledge (2). Thus, the aim of our study was to evaluate IPHs on FPCT findings after successful recanalization of MT, their clinical usefulness, and their ability to predict prognosis. The fate of IPH was also assessed.

\section{Methods}

\section{Patient selection}

We retrospectively reviewed 158 acute ischemic stroke patients who underwent endovascular treatment between January 2014 and November 2019. Twenty-one patients with posterior circulation were excluded. Inclusion criteria for patients were as follows: (I) age greater than or equal to 18 years; (II) pre-stroke modified Rankin Scale (mRS) score of less than or equal to 2; (III) presence of a large arterial

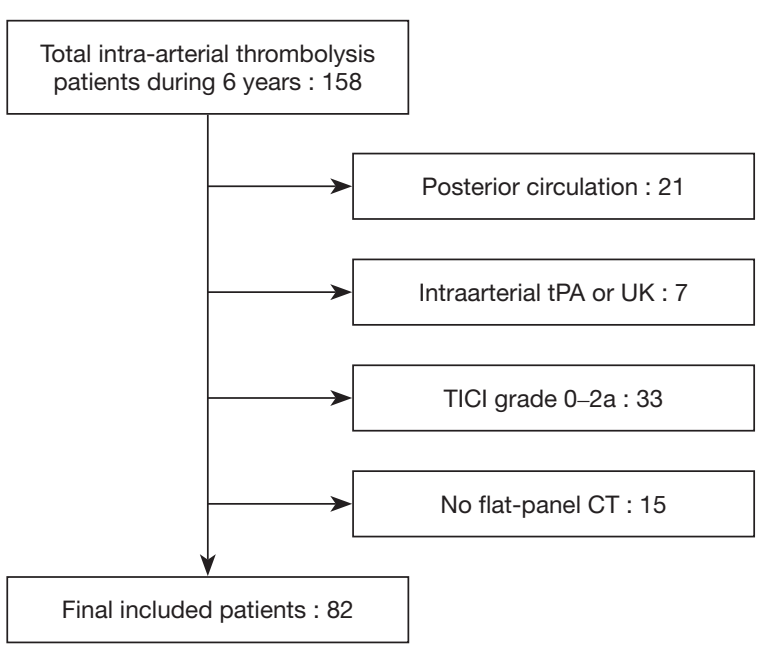

Figure 1 Inclusion criteria of this study. A total of 158 patients underwent intra-arterial thrombolysis due to acute ischemic stroke at our institute during the last 6 years. Among them, those with posterior circulation $(\mathrm{n}=21)$, those with intra-arterial thrombolysis without mechanical thrombectomy $(n=7)$, those with incomplete recanalization of TICI grade 0 to $2 \mathrm{a}(\mathrm{n}=35)$, and those who had no flat-panel computed tomography $(\mathrm{n}=15)$ were excluded. Finally, 82 patients were included in this study. CT, computed tomography; TICI, thrombolysis in cerebral infarction; $\mathrm{tPA}$, tissue plasminogen activator; UK, urokinase.

occlusion in anterior circulation [internal carotid artery (ICA) and/or middle cerebral artery (MCA)] as confirmed by CT angiography or digital subtracted angiography that was treated within 6 hours of stroke onset; and (IV) successful recanalization with thrombolysis in cerebral infarction (TICI) grade of $2 \mathrm{~b}-3$ (more than half of the occluded artery reperfusion). Patients were excluded from this study if they had been treated with intravenous tissue plasminogen activator (tPA) only or if they had achieved an incomplete recanalization (TICI grade of $0-2 a$ ) without FPCT after MT (11) (Figure 1). Posterior circulation infarction was excluded because it was not suitable for evaluation of post-procedural IPH pattern of striatal or cortical area. In addition, it shows a different clinical course. Patients with incomplete recanalization were also excluded mainly because the contrast media could not reach the infarcted area if the proximal artery was occluded. 

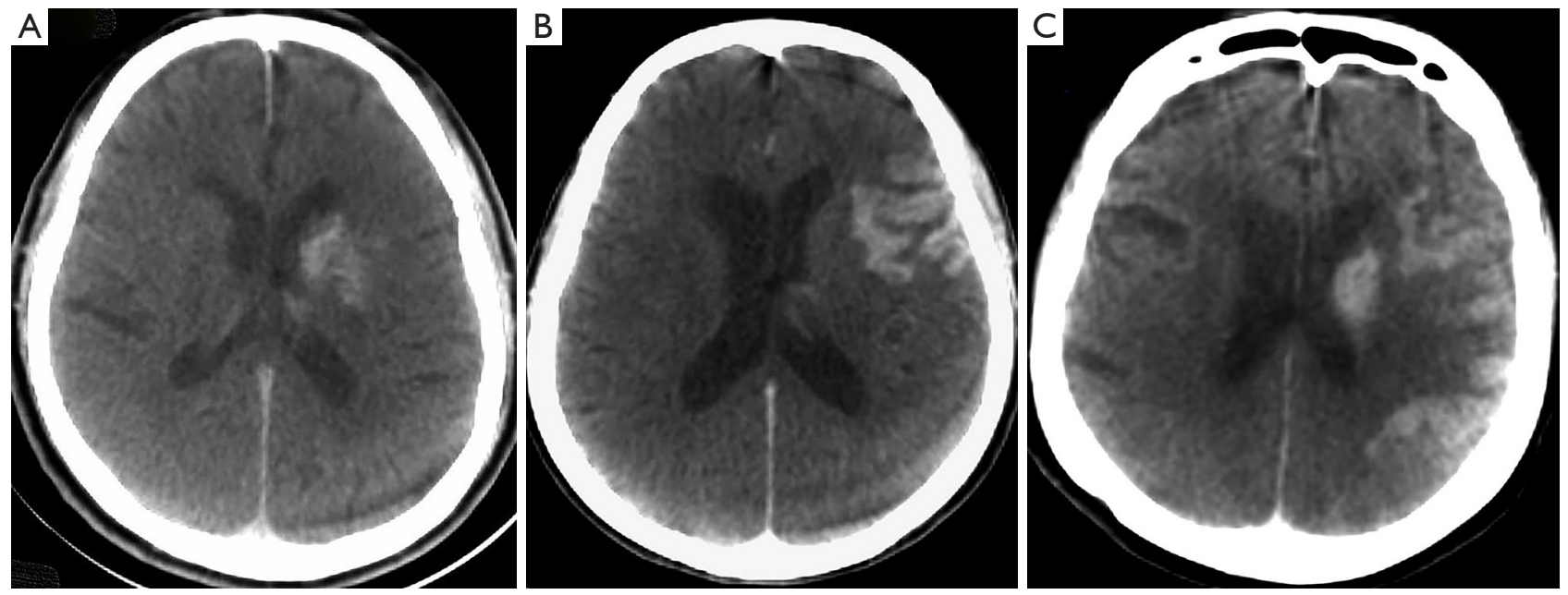

Figure 2 Patterns of contrast staining and localized subarachnoid hemorrhage on flat-panel computed tomography. (A) Striatal staining. (B) Regional cortical staining. (C) Striatal and cortical staining.

The procedure was performed with a stent-retriever [SolitaireTM FR revascularization device (ev3/Covidien, Irvine, CA, USA)] and a catheter for contact aspiration [Sofia intermediate catheter (MicroVention, Tustin, CA, USA) or a Penumbra reperfusion catheter (Penumbra, Alameda, CA, USA)]. This study was reviewed and approved by the Institutional Review Board of Kangbuk Samsung Hospital (IRB number: 2020-08-008). It was conducted in accordance with the Declaration of Helsinki (as revised in 2013). The requirement of informed consent was exempted due to its retrospective study design.

\section{Imaging interpretation}

An independent interventional neuroradiologist (MH Rho) and a vascular neurosurgeon (Y Chung) with more than five years of experience reviewed all imaging studies blinded to each other's interpretations. Any disagreements on imaging interpretation between these two primary readers were dissolved by another neurosurgeon (CE Hong). FPCT (Allura Xper FD 20 Flat detector with Xper-CT, Philips, Best, Netherlands) findings were reviewed immediately after MT in the angiography suite to identify if there was IPH. If IPH was identified, its patterns based on location were interpreted as follows: No IPH (pattern 0), IPH at the striatal area (pattern 1), cortical or subcortical IPH only in the extra-striatum area (pattern 2), striatal and cortical combined IPH in the ipsilateral anterior circulation territory (pattern 3) (Figure 2).
Post-procedural follow-up images were obtained according to the protocol of our institute except for cases with neurologic deterioration during management in the stroke unit. Follow-up magnetic resonance imaging (MRI) was performed at 48 hours after MT. For limited cases (6 patients, 9.8\%) which showed severe brain swelling with a poor neurological state, follow-up CT scans replaced MRI. Radiologic outcomes were categorized as follows: (I) recovery to normal structure, (II) persistent hyperattenuation and infarction without hemorrhage, (III) infarction with HT, and (IV) presence of intracerebral hemorrhage (ICH). Brain swelling with mid-line shifting was evaluated via the axial plane of the upper thalamus and the level of visualization of the septum pellucidum. Minimal brain swelling showing only sulci effacement without affecting a patient's prognosis was excluded. Severe brain swelling was defined as more than $10 \mathrm{~mm}$ of mid-line shifting (12).

\section{Clinical data}

Baseline demographic data including age, sex, lesion side, location of occluded artery, treatment modality and method, mean number of trials, and TICI grade were evaluated for all included patients. The time between the puncture and recanalization time as well as from $\mathrm{MT}$ to the first imaging follow-up MRI or CT were recorded. Medical records such as mRS scores at initial evaluation and at three-month postoperation were also analyzed. Good clinical outcomes were 
Table 1 Clinical characteristics according to flat-panel computed tomography findings of patients cohort

\begin{tabular}{|c|c|c|c|c|c|c|}
\hline Characteristics & All $(\mathrm{N}=82)$ & Non-IPH $(\mathrm{N}=48)$ & \multicolumn{3}{|c|}{ IPH $(\mathrm{N}=34)$} & $P$ value \\
\hline Mean age $^{\dagger}$, years & $71.8 \pm 12.3$ & $71.2 \pm 10.8$ & $70.6 \pm 13.3$ & $77.4 \pm 17.6$ & $71.8 \pm 13.3$ & 0.271 \\
\hline Male patients, $\mathrm{n}(\%)$ & $46(56.1)$ & $32(66.7)$ & $10(62.5)$ & $1(12.5)$ & $3(30.0)$ & 0.009 \\
\hline Right side, $\mathrm{n}(\%)$ & $39(47.6)$ & $25(52.1)$ & $8(50.0)$ & $1(12.5)$ & $5(50.0)$ & 0.211 \\
\hline$I V+I A$ & $47(57.3)$ & $27(56.3)$ & $8(50.0)$ & $5(62.5)$ & $7(70.0)$ & \\
\hline IA only & $35(42.7)$ & $21(43.8)$ & $8(50.0)$ & $3(37.5)$ & $3(30.0)$ & \\
\hline Treatment method, n (\%) & & & & & & 0.683 \\
\hline Stentreiver only & $74(90.2)$ & $42(87.5)$ & $14(87.5)$ & $8(100.0)$ & $10(100.0)$ & \\
\hline Mean procedural time (mins) ${ }^{\dagger}$ & $47.5 \pm 34.1$ & $48.0 \pm 39.3$ & $39.2 \pm 22.3$ & $55.0 \pm 36.4$ & $52.4 \pm 18.7$ & 0.253 \\
\hline Location of the occlusion, $n(\%)$ & & & & & & 0.039 \\
\hline ICA & $26(31.7)$ & $13(27.1)$ & $3(18.8)$ & $3(37.5)$ & $7(70.0)$ & \\
\hline MCA & $56(68.3)$ & $35(72.9)$ & $13(81.3)$ & $5(62.5)$ & $3(30.0)$ & \\
\hline $\mathrm{TICl}$ grade, $\mathrm{n}(\%)$ & & & & & & 0.342 \\
\hline $2 b$ & $24(29.3)$ & $11(22.9)$ & $6(37.5)$ & $4(50.0)$ & $3(30.0)$ & \\
\hline 3 & $58(70.7)$ & $37(77.1)$ & $10(62.5)$ & $4(50.0)$ & $7(70.0)$ & \\
\hline
\end{tabular}

${ }^{\dagger}$, other values. IA, intra-arterial; ICA, internal carotid artery; IPH, intra-parenchymal hyperattenuation; IV, intra-venous; MCA, middle cerebral artery; $\mathrm{TICl}$, thrombolysis in cerebral infarction.

defined as mRS scores of $0-2$.

\section{Statistical analysis}

Distributions of patients and baseline characteristics based on IPH patterns (non-IPH, striatal, cortical, or combined patterns) were compared using the KruskalWallis test for continuous variables and the Fisher's exact test for categorical variables. The Cochran-Armitage test was used to assess trends in hemorrhage incidence, brain swelling, and poor mRS scores based on IPH patterns. To analyze radiological and clinical outcomes at follow-up with univariate analysis, the Chi-square test or Fisher's exact test was used for categorical variables while Student's t-test or Mann-Whitney $U$ test was used for continuous variables, as appropriate. For variables with $\mathrm{P}<0.05$ in the univariate analyses, a multivariate logistic regression analysis was performed to determine independent risk factors. All statistical analyses were conducted using SPSS software version 24 (IBM Corp., Armonk, NY, USA).

\section{Results}

\section{Procedure-related variables and FPCT findings}

Table 1 shows similar clinical characteristics among IHP patterns except for sex and the location of the occlusion. Of 82 patients, 47 (57.3\%) underwent MT after intravenous administration of tPAs and 35 (42.7\%) underwent MT only. Seventy-four (90.2\%) patients were treated with stent-retrievers and $8(9.8 \%)$ patients were treated with combined mechanical/aspiration techniques. The mean procedure time from groin puncture to recanalization was $47.5( \pm 34.1) \mathrm{min}$ and the mean number of trials was 1.8 $( \pm 1.2)$. There were $26(31.7 \%)$ patients with ICA occlusion, including 4 patients who had an ICA bifurcation T-occlusion (carotid terminus occlusion which involved the ICA, MCA, and ACA) and 56 (68.3\%) patients with MCA occlusion, 
Table 2 Univariate analysis of variables with respect to radiologic and clinical outcomes

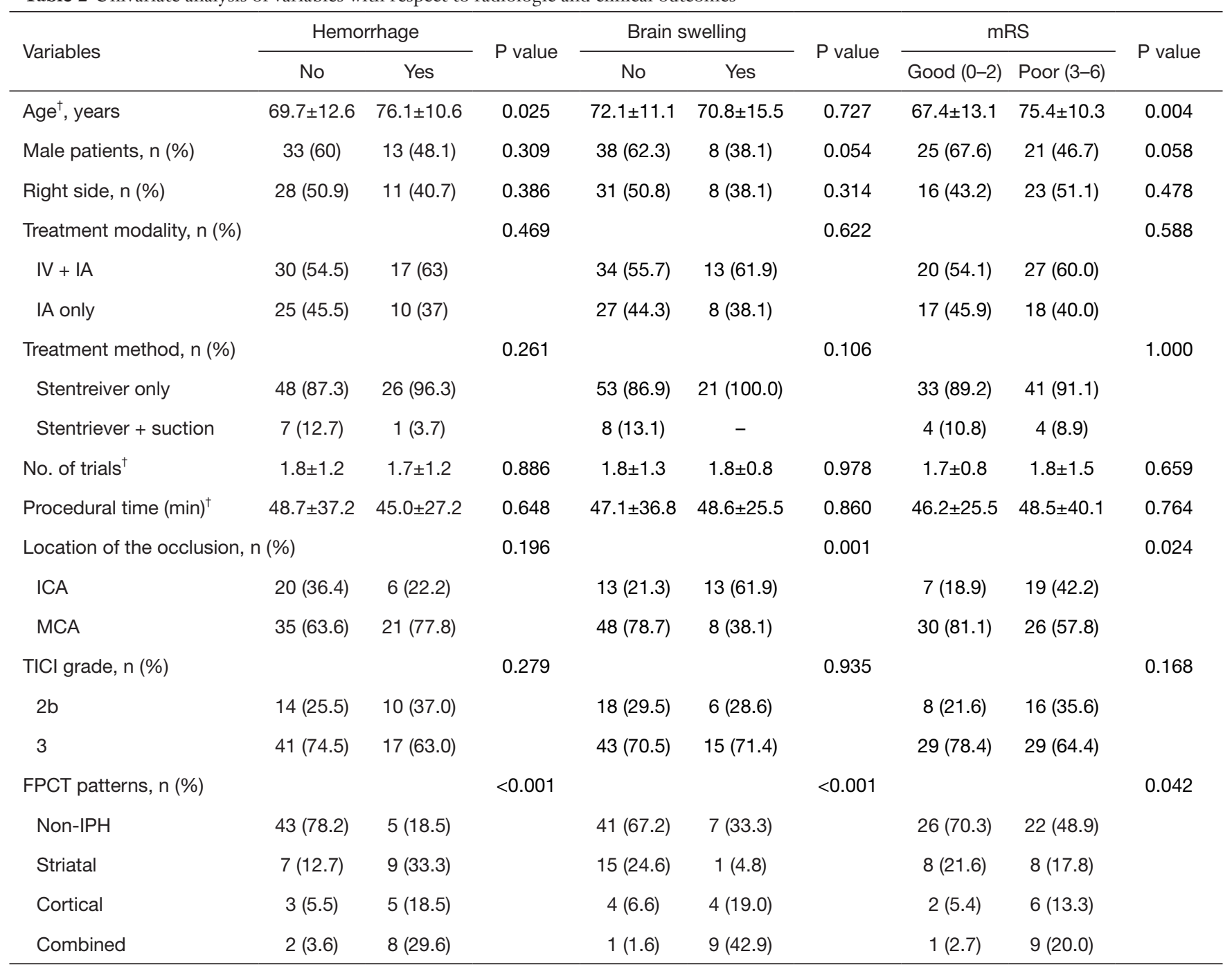

${ }^{\dagger}$, Other values, FPCT, flat-panel computed tomography; IA, intra-arterial; ICA, internal carotid artery; IPH, intra-parenchymal hyperattenuation; IV, intra-venous; MCA, middle cerebral artery; mRS, modified Rankin scale; TICl, thrombolysis in cerebral infarction.

including 10 patients who had an MCA M2 occlusion. IPH on FPCTs was seen in $34(41.5 \%)$ of 82 patients. Striatal pattern was observed in $16(19.5 \%)$ patients. Cortical or subcortical pattern was present in $8(9.8 \%)$ patients and combined pattern was found in $10(12.2 \%)$ patients (Table 1, Figure 2).

\section{Radiologic and clinical outcomes}

Most patients presented with post-procedural cerebral infarction (74 patients, 90.2\%). Among them, 47 (63.5\%) patients showed infarction only and 23 (31\%) patients progressed to HT (Table 2). Four (5.4\%) patients had ICH on follow-up images. Brain swelling accompanied by midline shifting was observed in $21(25.6 \%)$ patients. Six $(7.3 \%)$ underwent decompressive hemicraniectomy for severe brain edema. The average mid-line shifting was $8.8 \mathrm{~mm}$ (range: 3-29 mm). Eight (9.8\%) patients showed severe brain swelling with more than $10 \mathrm{~mm}$ of mid-line shifting.

Clinical outcomes demonstrated the following 3-month mRS score proportions: $\mathrm{mRS}$ score $=0$ ( 1 patient, $1.2 \%)$, mRS score $=1(20$ patients, $24.4 \%), \mathrm{mRS}$ score $=2(17$ patients, $20.7 \%)$, mRS score $=3$ (4 patients, $4.9 \%)$, mRS score $=4(14$ patients, $17.1 \%)$, mRS score $=5(17$ patients, $20.7 \%$ ), and $\mathrm{mRS}$ score $=6$ (9 patients, $11.0 \%)$. Thirtyseven $(45.1 \%)$ patients showed good clinical outcomes with 


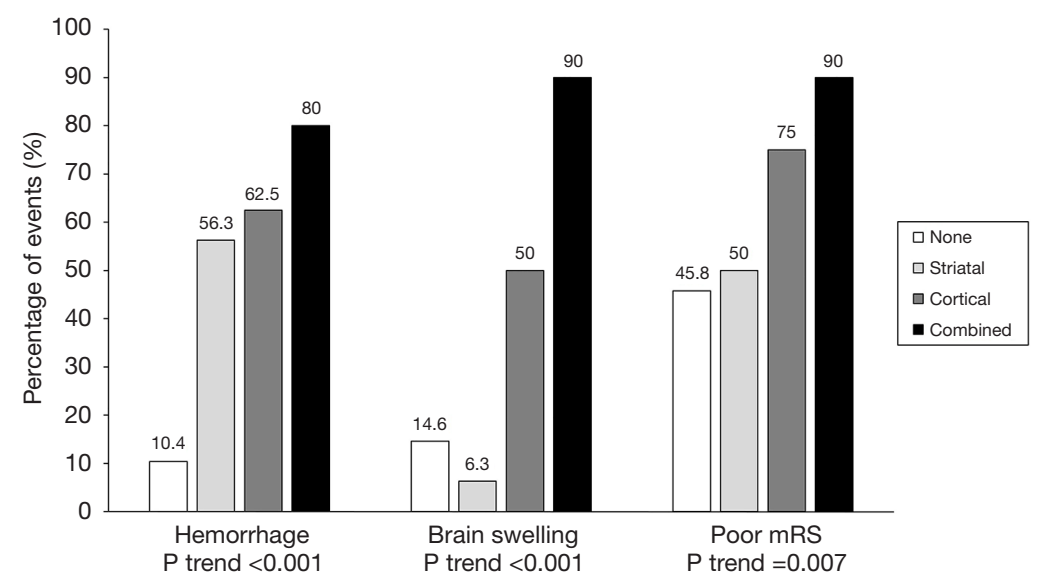

Figure 3 Associations between IPH patterns and radiologic or clinical outcomes (incidence of hemorrhage, brain swelling, and poor mRS score). P values were determined using the Cochran-Armitage trend test. IPH, intraparenchymal hyperattenuation; mRS, modified Rankin scale.

mRS scores of $0-2$.

\section{Outcomes according to staining pattern}

Radiologic and clinical outcomes were analyzed according to IPH patterns on FPCTs (Table 2). Those in the nonIPH group showed a relatively low incidence of HT, ICH, and brain swelling on follow-up images compared to the IPH group (8.3\% vs. $55.9 \%, 2.1 \%$ vs. $8.8 \%$, and $14.6 \%$ vs. $41.2 \%$, respectively) and better clinical outcomes based on $\mathrm{mRS}$ scores compared to the IPH group $(56.3 \%$ vs. $32.4 \%$ ). In the IPH group, hemorrhage (P trend $<0.001$ ), brain swelling ( $\mathrm{P}$ trend $<0.001)$, and poor $\mathrm{mRS}$ scores $(\mathrm{P}$ trend $=0.007)$ tended to increase in the presence of striatal, cortical, and combined patterns (Figure 3).

Univariate analysis was performed for four IPH patterns (non-IPH, striatal, cortical, and combined IPH pattern) in patients with hemorrhage (compared to those without hemorrhage), brain swelling (versus no swelling), and good mRS scores (versus poor mRS scores). Results are presented in Table 2. Hemorrhage, including HT and ICH $(\mathrm{P}<0.001)$, brain swelling $(\mathrm{P}<0.001)$, and poor $\mathrm{mRS}$ scores $(\mathrm{P}=0.042)$, presented differently among those with the four IPH patterns. There was a statistically significant difference in age according to the presence of hemorrhage and mRS score. The presence of brain swelling and mRS score depended on the location of the occlusion.

Multivariate logistic regression analysis was performed to confirm effects of radiologic outcomes, including hemorrhage, brain swelling, and functional outcomes
(Table 3). The striatal pattern independently predicted hemorrhage (OR: 13.26, $\mathrm{P}<0.01$ ). However, it showed no correlation with brain swelling or poor mRS score as compared to the non-IPH pattern. The cortical pattern significantly predicted hemorrhage (OR: 11.61, $\mathrm{P}=0.009)$ and brain swelling (OR: 5.94, $\mathrm{P}=0.039)$ without significant poor clinical outcome. However, the combined pattern foreshadowed poor clinical outcomes (OR: 10.58, P=0.046) with hemorrhage (OR: 45.34, $\mathrm{P}<0.001)$ and brain swelling (OR: $39.85, \mathrm{P}=0.001$ ). ICA occlusion was also related to brain swelling (OR: 4.13, $\mathrm{P}=0.034)$. Hemorrhage prevalence (OR: 1.06, $\mathrm{P}=0.033)$ and poor $\mathrm{mRS}$ score (OR: 1.07, $\mathrm{P}=0.006)$ were significantly influenced by age.

\section{Discussion}

\section{Intraparenchymal byperattenuation after mechanical thrombectomy}

Cerebral IPH after MT typically appears as an area showing hyperdensity. It is mostly confined to the grey matter (cortex and deep grey matter) and areas showing BBB breakdown secondary to ischemia with microvascular extravasation of contrast into the extracellular space $(1,9,13)$. The distribution of cerebral IPH correlates with the eventual volume of infarction. In other words, the pre-procedural infarct core plus any portions of penumbra which will go on to infarct despite treatment $(2,14)$. As such, IPH observed either on conventional multidetector CT or FPCT immediately following MT can provide prognostic 
Table 3 Multivariate logistic regression analysis of variables with respect to radiologic and clinical outcomes (odds ratio with $95 \%$ of confidence intervals)

\begin{tabular}{|c|c|c|c|c|c|c|}
\hline Variables & \multicolumn{2}{|c|}{ Hemorrhage } & \multicolumn{2}{|c|}{ Brain swelling } & \multicolumn{2}{|l|}{ Poor mRS } \\
\hline Age & $1.06(1.00-1.12)$ & 0.033 & - & - & $1.07(1.02-1.11)$ & 0.006 \\
\hline \multicolumn{7}{|c|}{ Location of the occlusion } \\
\hline ICA & - & - & $4.13(1.11-15.33)$ & 0.034 & $2.51(0.79-7.99)$ & 0.120 \\
\hline \multicolumn{7}{|c|}{ FPCT patterns } \\
\hline Non-IPH & Reference & - & Reference & - & Reference & - \\
\hline Striatal & $13.26(3.17-55.5)$ & $<0.001$ & $0.43(0.05-3.99)$ & 0.461 & $1.36(0.40-4.57)$ & 0.624 \\
\hline Cortical & $11.61(1.84-73.42)$ & 0.009 & $5.94(1.09-32.31)$ & 0.039 & $2.86(0.44-18.40)$ & 0.269 \\
\hline
\end{tabular}

FPCT, flat-panel computed tomography; ICA, internal carotid artery; IPH, intra-parenchymal hyperattenuation; MCA, middle cerebral artery; mRS, modified rankin scale.

information about the eventual volume of infarction. Therefore, IPH itself can provide clinical usefulness without directly measuring the core infarction size. Reported incidence of hyperdense lesions ranges from $23-84.2 \%$ in various patient cohorts $(1,3,9)$. It was $41.5 \%$ in the present study.

Takei et al. (15) have suggested that those with MT show a higher incidence of hyperdensity lesions due to higher recanalization rates and mechanical injury of intracranial vessels with the stent retriever. Typically, such contrast enhancement will clear within the first 19-24 hours after the procedure without hematoma $(4,16)$. Many studies have posited that IPH with densities exceeding 90 Hounsfield units is a hemorrhage. Some studies have reported that 19- to 24-hour follow-up CT is the only reliable method rather than using specific density thresholds to differentiate between contrast staining and hemorrhage $(4,17)$. Dualenergy CT can also accurately distinguish hyperdensity due to iodinated contrast from BBB disruption versus hyperdensity due to any ICH without any additional radiation $(3,18,19)$. Virtual non-contrast reconstructed images with dual-energy CT are also useful in the identification of acute ischemia (20). Distinguishing whether IPH is a contrast extravasation or a hemorrhage is important for deciding the following antithrombotic therapy and prognosis of the patient. Parrilla et al. (8) first reported that high-density lesions after endovascular treatment were not related to an increased risk of ICH.
However, results might be different depending on variables such as the definition of high-density lesions, the timing of the CT scan (immediately after MT or within 24 hours), and the differentiation between ICH and HT. Thereafter, several studies have reported that HT is more frequent in the contrast extravasation group than in patients without high density lesions $(29.7-68.4 \%$ vs. 0-39.5\%) (9,21,22). Results of the present study also showed significant differences between the IPH group and the non-IPH group: HT (55.9\% vs. $8.3 \%)$, ICH (8.8\% vs. $2.1 \%)$, brain swelling ( $41.2 \%$ vs. $14.6 \%)$, and poor mRS score $(67.6 \%$ vs. $45.8 \%)$. Furthermore, many studies have suggested that contrast extravasation itself is associated with other clinical indicators $(9,19,23)$. Neurotoxic effects or allergic reactions may occur due to contrast agents $(9,24)$.

There are some reports on the prognosis of IPH lesions by quantitative or qualitative analysis. Jang et al. (25) have categorized hyperdense lesions into four types: cortical, soft, metallic ( $>80$ Hounsfield units), and diffuse highdensity. They reported that all metallic density lesions, $50 \%$ of the diffuse type, $46.1 \%$ of the soft type, and $0 \%$ of the cortical type progressed to hemorrhage. Chen et al. (26) have reported that the presence of hyperdensity on FPCT can predict hemorrhagic transformation with a high sensitivity (96\%) and a high negative predictive value (98\%). Another study has shown a significant difference in symptomatic hemorrhage between the cortical contrast accumulation group and the non-cortical group (39.4\% 
vs. $4.3 \%, \mathrm{P}=0.003)$, suggesting that widespread contrast accumulation could be related to extensive reperfusion injury $(27,28)$. If covering about $20 \%$ or more of the hemisphere, the extent of contrast staining has a predictive value for brain edema or symptomatic hemorrhage in some studies $(15,17,23)$. Variations may exist depending on the modality of endovascular treatment (whether a stent retriever is used or not), recanalization rate, occlusion site, the quality or timing of post-procedural CT scans, amount of contrast agents, and the average time for fluoroscopy, all of which can influence the pattern or the amount of contrast staining. In addition, the successful recanalization rate is lower in posterior circulation (vertebrobasilar) stroke (50\%) than in ICA or MCA stroke (82-89\%) (29). Therefore, we established anterior circulation and mechanical thrombectomy with TICI grade $2 \mathrm{~b}$ and 3 as a relatively narrow inclusion criteria for this study and tried to analyze the clinical meaning of hyperattenuation. In our study, IPH was significantly associated with an increased frequency of hemorrhagic complications, brain swelling, and poor clinical outcomes. One possible reason for these results might be abundant collateral circulation, which is one of the most important factors in predicting prognosis. Further analysis with pre-procedural perfusion information will strengthen the meaning of IPH based on hemodynamics.

\section{Different outcomes according to IPH patterns}

When determining the clinical usefulness of IPH, both qualitative and quantitative analyses of anatomical location are important. FPCT is limited to quantitative or density threshold analysis of IPH. However, it is sufficient for hyperattenuation pattern analysis. Confined contrast staining in the striatum may not predict clinically malignant hemorrhage since rapid recanalization or robust collateral is possible. Hence, the functional outcome is expected to better for those with striatal IPH patterns. On the other hand, cortical or subcortical patterns reflect widespread BBB breakdown and reperfusion injury with possible involvement at the eloquent-area associated with initial ischemic insult due to poor collaterals (27). Previous research has revealed that the prevalence of symptomatic hemorrhage is different according to the site of contrast accumulation in conventional non-contrast CT scans (27). In our multivariate analysis, the presence of a cortical pattern was a stronger predictor for brain swelling than that of a striatal pattern (OR of $5.94 v s$. OR of 0.43 ).

Our categorization of the IPH pattern was based on the location by FPCT. This also reflects the concept of core infarction size. In fact, striatal pattern qualification suggested MCA M1 occlusion and good collateral of the distal MCA territory and the combined pattern on FPCT scans suggested extensive infarction involving the IPH area with poor distal collaterals. Therefore, patients with this presentation can be predicted to have a poor clinical prognosis. In our multivariate analysis, the presence of a combined pattern was the strongest predictor for hemorrhage (OR: 45.34, $\mathrm{P}<0.001$ ), brain swelling (OR: $39.85, \mathrm{P}=0.001$ ), and poor $\mathrm{mRS}$ score (OR: $10.58, \mathrm{P}=0.046$ ), showing significant differences compared to other patterns (Table 3). Figure 4 illustrates the evolution of the IPH area. Although patients who presented with the striatal pattern proceeded to HT, brain swelling rarely occurred, whereas patients with a combined pattern proceeded to malignant brain swelling with poor clinical outcomes.

\section{The role of flat-panel CT after mechanical thrombectomy}

Recently, several studies have reported the usefulness of dual-energy CT for the differentiation of hemorrhage and contrast extravasation $(3,18,30)$. Although FPCT has disadvantages compared to dual-energy CT in this respect, it is sufficient and still important for the assessment of hemorrhage after MT for acute ischemic stroke. Dual-energy CT is not yet a standard method. Post-procedural FPCT is a simple, reliable, and rapid tool for prompt follow-up after MT in the angiography suite without additional patient transport $(2,15,31)$. Rouchaud et al. (31) and Chen et al. (26) have reported the performance of FPCT for detecting risk factors for the prediction of hemorrhagic complications with a high positive predictive value $(84.2 \%$ and $72 \%$, respectively) and a high negative predictive value $(92.0 \%$ and $98 \%$, respectively). Furthermore, regardless of the origin of IPH (from BBB breakdown or contrast extravasation), IPH is associated with a higher risk for HT and poorer clinical outcomes. FPCT is a cost-effective and important modality that provides important information for predicting the prognosis after MT. The C-arm mounted flat-panel detector immediately after completion of MT on the angiography table simply allows the detection of hemorrhagic lesion by non-radiologists in an acute setting (31). One of the most important findings of this study was that the IPH pattern, which could be easily identified in FPCT, could provide important information for the prediction of clinical outcomes. In acute ischemic stroke, even after successful recanalization, early evaluation is 

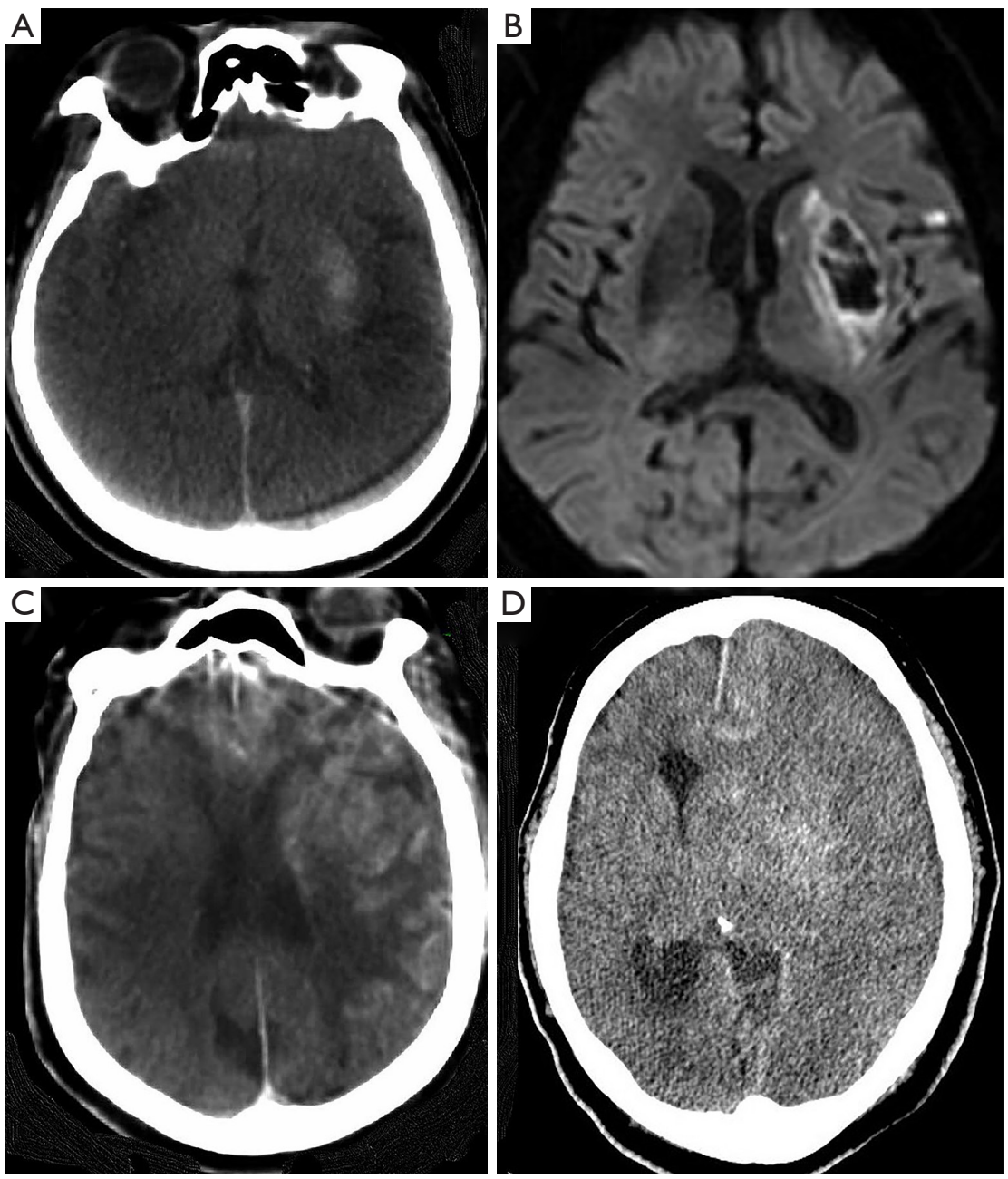

Figure 4 Evolution of the contrast staining area. (A) FPCT just after successful recanalization of the occlusion of the left distal M1 showing striatal stating; (B) follow-up diffusion-weighted imaging revealing lower signal intensity hemorrhagic transformation in the striatal infarcted area; (C) FPCT axial image just after successful recanalization of the occlusion of the left distal intra-carotid artery (carotid-T) showing striatal and diffuse cortical staining; (D) follow-up CT demonstrating hemorrhagic transformation and marked brain swelling at one day after recanalization. She died the next day. FPCT, flat-panel computed tomography.

important because hemorrhagic complications related to anticoagulation and reperfusion injury can occur dynamically immediately after the procedure. It has the advantage of preparing for hemorrhagic complications with prompt and simple evaluation using FPCT scan in an angiography suite.

\section{Limitations}

Several limitations exist in this study. First, this study had a retrospective design from a single center with a relatively small number of patients, resulting in the potential risk of selection bias. Nevertheless, we included anterior circulation patients with only successful recanalization (TICI $2 \mathrm{~b}$ and 3 ) after mechanical thrombectomy to make the treatment modality homogeneous. Another debatable aspect was the quantitative nature of IPH area analysis. We only evaluated qualitative IPH patterns while focal cortical high-density area was excluded. Next, despite our routine follow-up imaging protocol, there was heterogeneity in imaging modality and time due to the inclusion of neurological deterioration cases, which required a decompressive craniotomy. Finally, the injected volume of contrast media, which could be a factor affecting 
IPH, could not be controlled during the procedure. Future experimental studies with large populations are needed to further evaluate the pathophysiological findings of this study.

\section{Conclusions}

According to IPH patterns, poor radiologic and functional outcomes tended to increase significantly. Those with a striatal pattern showed relatively good clinical outcomes despite significantly higher hemorrhagic complications. Cortical IPH patterns independently predicted higher postprocedural hemorrhage or brain swelling. The combined pattern is a strong predictor for both radiologic outcomes and poor clinical outcomes. FPCT is an effective and useful modality immediately after MT for predicting both radiologic outcomes and clinical prognosis.

\section{Acknowledgments}

Funding: None.

\section{Footnote}

Conflicts of Interest: All authors have completed the ICMJE uniform disclosure form (available at https://dx.doi. org/10.21037/qims-21-322). The authors have no conflicts of interest relevant to this study to disclose.

Ethical Statement: The authors are accountable for all aspects of the work in ensuring that questions related to the accuracy or integrity of any part of the work are appropriately investigated and resolved. This study was reviewed and approved by the Institutional Review Board of Kangbuk Samsung Hospital (IRB number: 2020-08-008). It was conducted in accordance with the Declaration of Helsinki (as revised in 2013). The requirement of informed consent was exempted due to its retrospective study design.

Open Access Statement: This is an Open Access article distributed in accordance with the Creative Commons Attribution-NonCommercial-NoDerivs 4.0 International License (CC BY-NC-ND 4.0), which permits the noncommercial replication and distribution of the article with the strict proviso that no changes or edits are made and the original work is properly cited (including links to both the formal publication through the relevant DOI and the license). See: https://creativecommons.org/licenses/by-nc-nd/4.0/.

\section{References}

1. Lummel N, Schulte-Altedorneburg G, Bernau C, Pfefferkorn T, Patzig M, Janssen H, Opherk C, Brückmann H, Linn J. Hyperattenuated intracerebral lesions after mechanical recanalization in acute stroke. AJNR Am J Neuroradiol 2014;35:345-51.

2. Schneider T, Mahraun T, Schroeder J, Frolich A, Hoelter P, Wagner M, Darcourt J, Cognard C, Bonafe A, Fiehler J, Siemonsen S, Buhk JH. Intraparenchymal Hyperattenuations on Flat-Panel CT Directly After Mechanical Thrombectomy are Restricted to the Initial Infarct Core on Diffusion-Weighted Imaging. Clin Neuroradiol 2018;28:91-7.

3. Phan CM, Yoo AJ, Hirsch JA, Nogueira RG, Gupta R. Differentiation of hemorrhage from iodinated contrast in different intracranial compartments using dual-energy head CT. AJNR Am J Neuroradiol 2012;33:1088-94.

4. Dekeyzer S, Nikoubashman O, Lutin B, De Groote J, Vancaester E, De Blauwe S, Hemelsoet D, Wiesmann M, Defreyne L. Distinction between contrast staining and hemorrhage after endovascular stroke treatment: one CT is not enough. J Neurointerv Surg 2017;9:394-8.

5. Yedavalli V, Sammet S. Contrast Extravasation versus Hemorrhage after Thrombectomy in Patients with Acute Stroke. J Neuroimaging 2017;27:570-6.

6. Payabvash S, Qureshi MH, Khan SM, Khan M, Majidi S, Pawar S, Qureshi AI. Differentiating intraparenchymal hemorrhage from contrast extravasation on postprocedural noncontrast $\mathrm{CT}$ scan in acute ischemic stroke patients undergoing endovascular treatment. Neuroradiology 2014;56:737-44.

7. Uchiyama Y, Abe T, Tanaka N, Kojima K, Uchida M, Hirohata M, Hayabuchi N. Factors contributing to bloodbrain barrier disruption following intracarotid injection of nonionic iodinated contrast medium for cerebral angiography: experimental study in rabbits. Radiat Med 2006;24:321-6.

8. Parrilla G, Garcia-Villalba B, Espinosa de Rueda M, Zamarro J, Carrion E, Hernandez-Fernandez F, Martin J, Hernandez-Clares R, Morales A, Moreno A. Hemorrhage/ contrast staining areas after mechanical intra-arterial thrombectomy in acute ischemic stroke: imaging findings and clinical significance. AJNR Am J Neuroradiol 2012;33:1791-6.

9. Chen Z, Zhang Y, Su Y, Sun Y, He Y, Chen H. Contrast Extravasation is Predictive of Poor Clinical Outcomes in Patients Undergoing Endovascular Therapy for Acute 
Ischemic Stroke in the Anterior Circulation. J Stroke Cerebrovasc Dis 2020;29:104494.

10. Cabral FB, Castro-Afonso LH, Nakiri GS, Monsignore LM, Fábio S, Dos Santos AC, Pontes-Neto OM, Abud DG. Hyper-attenuating brain lesions on CT after ischemic stroke and thrombectomy are associated with final brain infarction. Interv Neuroradiol 2017;23:594-600.

11. Zaidat OO, Yoo AJ, Khatri P, Tomsick TA, von Kummer $\mathrm{R}$, Saver JL, et al. Recommendations on angiographic revascularization grading standards for acute ischemic stroke: a consensus statement. Stroke 2013;44:2650-63.

12. Puffer RC, Yue JK, Mesley M, Billigen JB, Sharpless J, Fetzick AL, Puccio A, Diaz-Arrastia R, Okonkwo DO. Long-term outcome in traumatic brain injury patients with midline shift: a secondary analysis of the Phase 3 COBRIT clinical trial. J Neurosurg 2018;131:596-603.

13. Kim JT, Heo SH, Cho BH, Choi SM, Lee SH, Park MS, Yoon W, Cho KH. Hyperdensity on non-contrast CT immediately after intra-arterial revascularization. J Neurol 2012;259:936-43.

14. Amans MR, Cooke DL, Vella M, Dowd CF, Halbach VV, Higashida RT, Hetts SW. Contrast staining on CT after DSA in ischemic stroke patients progresses to infarction and rarely hemorrhages. Interv Neuroradiol 2014;20:106-15.

15. Takei J, Irie K, Tanaka T, Okuno K, Hasegawa I, Shimoyama T, Yaguchi H, Hatano K, Maruyama F, Yamamoto Y, Tochigi S, Hasegawa Y, Murayama Y. Evaluation of the CT High-density Area after Endovascular Treatment for Acute Ischemic Stroke. J Neuroendovascular Ther 2017;11:227-34.

16. Yoon W, Seo JJ, Kim JK, Cho KH, Park JG, Kang HK. Contrast enhancement and contrast extravasation on computed tomography after intra-arterial thrombolysis in patients with acute ischemic stroke. Stroke 2004;35:876-81.

17. Xu C, Zhou Y, Zhang R, Chen Z, Zhong W, Gong X, Ding X, Lou M. Metallic Hyperdensity Sign on Noncontrast CT Immediately after Mechanical Thrombectomy Predicts Parenchymal Hemorrhage in Patients with Acute Large-Artery Occlusion. AJNR Am J Neuroradiol 2019;40:661-7.

18. Morhard D, Ertl L, Gerdsmeier-Petz W, Ertl-Wagner B, Schulte-Altedorneburg G. Dual-energy CT immediately after endovascular stroke intervention: prognostic implications. Cardiovasc Intervent Radiol 2014;37:1171-8.

19. Renú A, Amaro S, Laredo C, Roman LS, Llull L, Lopez A, Urra X, Blasco J, Oleaga L, Chamorro A. Relevance of blood-brain barrier disruption after endovascular treatment of ischemic stroke: dual-energy computed tomographic study. Stroke 2015;46:673-9.

20. Gariani J, Cuvinciuc V, Courvoisier D, Krauss B, Mendes Pereira V, Sztajzel R, Lovblad KO, Vargas MI. Diagnosis of acute ischemia using dual energy CT after mechanical thrombectomy. J Neurointerv Surg 2016;8:996-1000.

21. Nakano S, Iseda T, Kawano H, Yoneyama T, Ikeda T, Wakisaka S. Parenchymal hyperdensity on computed tomography after intra-arterial reperfusion therapy for acute middle cerebral artery occlusion: incidence and clinical significance. Stroke 2001;32:2042-8.

22. Chen WH, Yi TY, Wu YM, Zhang MF, Lin DL, Lin XH. Parenchymal hyperdensity on C-arm CT images after endovascular therapy for acute ischaemic stroke predicts a poor prognosis. Clin Radiol 2019;74:399-404.

23. Song SY, Ahn SY, Rhee JJ, Lee JW, Hur JW, Lee HK. Extent of Contrast Enhancement on NonEnhanced Computed Tomography after Intra-Arterial Thrombectomy for Acute Infarction on Anterior Circulation: As a Predictive Value for Malignant Brain Edema. J Korean Neurosurg Soc 2015;58:321-7.

24. Gomi N. Vasoconstriction by angiographic contrast media in isolated canine arteries. Br J Radiol 1992;65:961-7.

25. Jang YM, Lee DH, Kim HS, Ryu CW, Lee JH, Choi CG, Kim SJ, Suh DC. The fate of high-density lesions on the non-contrast $\mathrm{CT}$ obtained immediately after intra-arterial thrombolysis in ischemic stroke patients. Korean J Radiol 2006;7:221-8

26. Chen L, Xu Y, Shen R, Sun J, Zhang X, Zhang Q, Wang F. Flat Panel CT Scanning Is Helpful in Predicting Hemorrhagic Transformation in Acute Ischemic Stroke Patients Undergoing Endovascular Thrombectomy. Biomed Res Int 2021;2021:5527101.

27. Kim JM, Park KY, Lee WJ, Byun JS, Kim JK, Park MS, Ahn SW, Shin HW. The cortical contrast accumulation from brain computed tomography after endovascular treatment predicts symptomatic hemorrhage. Eur J Neurol 2015;22:1453-8.

28. Zhu T, Ren L, Zhang L, Shao Y, Wan L, Li Y, Liang D, Zheng H, Liu X, Zhang N. Comparison of plaque characteristics of small and large subcortical infarctions in the middle cerebral artery territory using high-resolution magnetic resonance vessel wall imaging. Quant Imaging Med Surg 2021;11:57-66.

29. Vavrova J, Koznar B, Peisker T, Vasko P, Rohac F, Sulzenko J, Kroupa J, Stetkarova I, Mengozzi L, Petras M, Widimsky P. Long-term outcomes of thrombectomy 
for acute ischaemic stroke by occluded artery and stroke aetiology: a PRAGUE-16 substudy. EuroIntervention 2021;17:e169-77.

30. Gupta R, Phan CM, Leidecker C, Brady TJ, Hirsch JA, Nogueira RG, Yoo AJ. Evaluation of dual-energy CT for differentiating intracerebral hemorrhage from iodinated contrast material staining. Radiology 2010;257:205-11.

31. Rouchaud A, Pistocchi S, Blanc R, Engrand N, Bartolini B, Piotin M. Predictive value of flat-panel CT for haemorrhagic transformations in patients with acute stroke treated with thrombectomy. J Neurointerv Surg 2014;6:139-43.

Cite this article as: Chung $\mathrm{Y}$, Bae Y, Hong CE, Won YS, Baek JH, Chung PW, Kim MS, Rho MH. Hyperattenuations on flat-panel computed tomography after successful recanalization of mechanical thrombectomy for anterior circulation occlusion. Quant Imaging Med Surg 2022;12(2):1051-1062. doi: 10.21037/ qims-21-322 